\title{
GSM Digital Cellular Telephone System A Case Study of Encryption Algorithms
}

\author{
Tony Smith \\ Deputy Director \\ Centre for Telecommunications Information Networking, \\ University of Adelaide, 33 Queen Street, Thebarton, South Australia, \\ 5031. Tel: +61-8-3033222 Fax: +61-8-3034405 \\ Email: tsmith@ctin.adelaide.edu.au
}

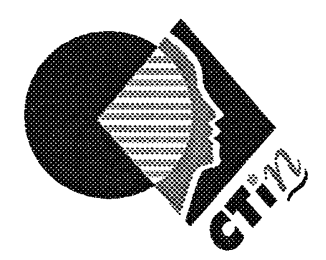

\section{Centre for Telecommunications Information Networking}

\begin{abstract}
:
There have been many reports in the newspapers of eavesdropping and cloning of telephones in the analogue network. However is the digital network free from these attacks? Security was seen as a high priority and was addressed in the development of GSM, this paper looks at fraud in GSM and shows how it has not been eliminated, in fact due to the flexibility and the new services it introduced GSM has created new forms of fraud. GSM includes three algorithms for the purposes of Authentication, Air Interface Encryption and Key Delivery (A3, A5 and A8). The paper looks at the history of the algorithms and the different versions.
\end{abstract}

\section{Keywords}

Global system for Mobiles, GSM, A3, A5, A8, Encryption, Fraud, 


\section{INTRODUCTION}

GSM mobile voice networks are being deployed extensively throughout the world with 140 or more networks now in existence. It must be noted no matter what the GSM community would like the reality is GSM will not become the exclusive mobile network technology but will share the world market with many other technologies. GSM should not be considered as a competitor but as a platform to understand the issues that will confront a mobile data network. The important underlying issue is the move from fixed to mobile communications, be it voice or data and the problems this brings. Interception of the radio link without knowledge is the first issue followed by cloning and lack of knowledge of the calling parties location. Billing and charging problems increase and is similar to the credit card industry where authentication of a person's identity is required. In a fixed network the location of the wall socket and terminal equipment bears a direct relationship to the creditor. GSM has also had extensive experience due to its deployment in many of countries, handsets are being sold extensively into the consumer market whereas many mobile data products are still penetrating the lower risk corporate market.

In designing GSM an assessment of risk was used to assist in the work of the security expert group. The first objective was to produce a robust authentication mechanism, the second to make the radio path as secure as the fixed line telephone network (PSTN) to preserve the caller's anonymity and privacy. The third objective was to prevent one operator from compromising the security of another network either by accident or due to pressures from competition. All of this must be met with penalty using a cost effective solution.

\section{EXISTING ANALOGUE SYSTEMS}

Analogue mobile telephone networks are known for a third party to eavesdrop on a conversation, with many notable examples worldwide, another problem is handset cloning for the purposes of committing fraud. Eavesdropping is common, all that is required is a cheap scanner, that along with some spare time, a tape recorder and you are well on your way to obtaining your 15 minutes of fame. Cloning is not as simple but is achieved by capturing mobile handset codes which are sent on an AMPS and TACS system in clear mode every time a handset registers or makes a call. Devices readily available will capture these and then put these into a modified handsets for use. One technique called 'Tumbling' uses each cloned code once before the next set are installed automatically into the handset. This method builds on the knowledge that a typical mobile customer doesn't check their account on a call by call basis and so one extra call goes unnoticed. Even if it is noticed the problem is how to prove to the network operator you didn't make that call and then who is responsible for fraudulent calls. 


\section{THE ROLE OF STANDARDS IN GSM DEVELOPMENT}

GSM has had a somewhat unique development life cycle as it is one of the success stories of a system developed under a standards environment. Typically standards bodies are perceived as being slow and conservative, some steps behind the industry leaders who develop and market proprietary solutions. GSM provides a different model to that typically seen in the IT industry as it is cooperative approach to systems development. GSM began in the early 80's with the realisation of the need for a pan European mobile telephone system, it built on the experience of the NMT analogue cellular system which covered the Scandinavian countries. A FrenchGerman agreement saw the standards organisation CEPT form the Groupe Speciale Mobile in 1982. Digital was thought to be the solution with a data rate of $16 \mathrm{kbps}$ or something close to it and to resolve the principles of operation detailed technical simulations on spectrum efficiency, delays and queuing were performed from 1984 to 1986 . During 1985 France and Germany joined to build prototypes based on four alternatives and in 1987 the official decision was made on the preferred solution. A harmonised Europe issued an EC Directive establishing the European Telecommunications Standards Institute (ETSI) in 1988 with the work of CEPT being transferred to ETSI in 1989. ETSI Special Mobile Group (SMG) is the committee which ratifies work from its nine technical committees (SMG1 to SMG9), these are supported by a full time project team (PT12) which manages the ongoing development.

The important lesson from GSM is the how the participants with different goals and business priorities took a strategic position that although they may have preferred their own solutions they were prepared to put the alternatives to a committee vote and adopt the result. They saw the benefits stemming from commonality and standardisation as being far more important than owning the Intellectual Property. The benefit coming rather than proprietary solution is in the profits derived from mass production for a world market.

GSM developed in the same time frame as the rising demand for mobile telephone services, solving the problem of roaming across Europe has provided a solution for roaming on a 'global' basis. Today as the system is reaching what many think is development maturity in Europe it is going through extensive enhancement particularly with the introduction of enhanced data services under the title of General Packet Radio Service (GPRS) which when implemented will provide a connectionless packet data service to mobiles. Equally the migration of the technology into the US market has provided stimulation for the rapid introduction of enhancements using improved handset processing capacity. The US has seen the introduction of a new voice codec which provides improved voice quality, the timing of the release of new spectrum $(1900 \mathrm{Mhz})$ has motivated GSM to finalise the enhanced features under Phase 2 of the standard. 


\section{SIM CARD \& ISO STANDARDS}

\section{Role of the SIM Card in GSM}

The Subscriber Identity Module (SIM) contains ALL the information relevant to the customer and so the SIM can be swapped from one handset to another for the purpose of handset maintenance. One initial concept was to be able to issue a SIM card to a person for them to use in any vehicle, private or company, taxi etc. This would allow people to remove the card from their low power pocket portable and insert it into their higher power car mounted mobile. It is interesting to see how an original concept failed to materialise as networks were being built for high traffic levels resulting in relatively small cell sizes which work very well with pocket portable units. This eliminated the need for high power mobiles, where as vehicle adaptor kits providing battery charging and handsfree operation are popular.

The SIM was a marketing feature of GSM but is neither provides a benefit or problem for customers, what it has become is the system component that provides the network operator customer relationship. Customers can roam onto a GSM network elsewhere in the world and the costs are billed by their home operator, the home operator provides all the authentication services as they are held responsible for the intercarrier charges. Also GSM does not divulge to the other network operators secret information about their customers.

\section{SIM Design}

The SIM is built to ISO standard 7816 for contact type smartcards with the data elements and communication protocols under the ISO framework. To achieve this a GSM directory was established separate from the established Telecom directory, this contains information about preferred networks, prohibited networks, active services etc. It also provides the mechanism to communicate the challenge (RAND) and response (SRES) to and from the card for authentication.

\section{SIM Card Manufacture and Personalisation}

SIMs are manufactured as blank cards although the operator determines the algorithms which are built into the mask of the SIM memory. Each operator can load the customer specific data 'personalise the SIM', however the most common method is to outsource this to the SIM card manufacturer. A third and more expensive method is to personalise the cards at the point of sale by using a system which communicates securely with the operator's Authentication Centre.

\section{FRAUD}

\subsection{Impacts of Fraud}

Fraud in cellular is being committed as a organised business, one European operator published figures that showed the number of fraud events being constant as their network grew from 50,000 to 150,000 customers. They didn't see the fraud rate increase in proportion to their 
customers base and the conclusion is that vast majority of customers are honest and methods to limit fraud will impact on all trustworthy customers.

Fraud has a major impact on all sides of the industry it can't be ignored as being small to insignificant. It WILL occur and its a matter of managing it to keep it to an acceptable level. The credit card industry manages it at about $1.5 \%$ of turnover and are working on ways to reduce it further. It will impact customers, the operators revenue and network performance as well as the industry by reducing growth.

The question an operator should ask themselves is; why should I bother focussing on fraud? Why bother committing scarce human resources to fraud control and why spend money on fraud control?

\section{Impact On Customers}

Customers who end up with mystery calls on their accounts become dissatisfied. In the majority of cases these calls go unnoticed as cloning normally only places one additional call on each account. As most people don't check their accounts down to the transaction level these extra costs go unnoticed.

However if the additional call is noticed how do you convince the operator that the call wasn't made by yourself. This leads to dissatisfaction with the service being provided, it usually involves a number of people at the customer service centre and it's common to see the customer arguing with the operator.

Another aspect of fraud is that being an organised business it occurs in specific locations in large quantities, this leads to increased network traffic and from the customers perspective is seen as poor service due to network blocking.

\section{Impact On The Operator}

Financially, fraud can exceed $2 \%$ of turnover with $3 \%$ being easily achieved before it is noticed. Due to the large network capital investment a fraud level of 1-2\% of turnover can easily equate to $5-10 \%$ of profit. With future competition resulting in lower margins and with the average revenue per customer reducing as sales move from the corporate to the consumer market the effect of fraud will become more significant in reducing profit margins.

Fraud also increases the operational costs of customer service centres. Every account enquiry takes a significant amount of time to resolve and often require multiple calls are required to resolve a dispute.

Customer dissatisfaction not only leads to additional churn, which is already excessively high but it also effects sales as personal recommendation is a significant part of the sales effort, we also have found bad publicity takes some 2 years to be negated.

Network loading is often affected by fraudulent traffic and is often see on a localised basis. Localised blocking not only affects customers but also sees the operator responds by installing more base stations, the associated costs which will never be recovered. A UK operator 
temporarily blocked international call forwarding from part of their London network and found that the majority of the traffic disappeared, this load was seen to be fraudulent.

\section{Industry Impact}

Fraud reduces growth of the industry, i.e. it affects people who are potential customers. The consumer market is particularly sensitive to price and have little margin to absorb fraudulent charges, therefore if they have the perception they will incur fraudulent charges they refrain from becoming customers.

In the US analogue networks where methods have been introduced to limit fraud they have introduced barriers to the use of services. e.g. to make a long distance call the customer calls an operator and uses a credit card. Due to the inconvenience, these measures inhibit the use of the mobile phones, but they do provide an opportunity for new networks to compete for customers without price being the sole differentiator.

Law enforcement agencies have been known to influence the industry particularly when it is associated with organised crime. In Australia the launch of GSM was delayed due to the issues relating to call interception and in Pakistan the GSM network was temporarily shut down soon after launch due to concerns about the lack of the law enforcement agencies ability to intercept calls.

\subsection{Fraud Types}

Fraudsters have no intention for paying for the service they are either using themselves or reselling for their own profit. They arrange to have the airtime charged to a false account, to someone else's existing account or even onto a non existent account.

One method of classifying fraud is Technical, Subscription and Internal.

- Technical fraud is about exploiting weakness of the technology or the network. A simple example, network fraud could be achieved by entering the network by a modem connected to the Mobile Swithing Centre (MSC) for remote maintenance. More common forms use diversion, cloning of identity codes etc.

- Subscription fraud is the most common method of fraud and is the classic fraud committed within the fixed telephone network. It is also the same fraud experienced by the Credit Card industry and so operators can use a number of existing strategies to reduce this form of attack.

- Internal fraud is less obvious but can account for a significant proportion of fraud losses particularly as other forms are reduced. 


\subsection{Fraud Examples}

\section{Subscription Fraud}

Subscription fraud relies on providing false identity, this is easier to achieve in a mobile network where as in a fixed network the service requires a wall socket and terminal equipment which is related to a physical location or building and therefore a person.

It can be significantly reduced by asking for proof of identity at the point of sale. This needs to be managed carefully as these documents can be stolen or altered. If you accept electricity or gas accounts for proof then it must be understood that these are easily stolen and are not reliable. There is an industry built around producing false documents and any fraud management team should learn how to obtain these documents so they know how to identify them. It is best to only use documents that theft can be verified in real time e.g. Cheques, Credit Cards are good examples.

Once the persons identity is known, credit checking can be added as these agencies have already established multiple sources of information.

The third method is to limit the risk by building a relationship with the customer. Letting the customer build a credit history is a good method as few fraudsters will pay accounts for 6 to 12 months and then commit fraud for a one or two month period. Credit limits can be built into the network as an Intelligent Network (IN) function as this will block outgoing calls until the account is paid. Deposits, or money in advance also deter fraudsters. In Recent times payment in advance systems are being introduced, these can be network based, a variation of the credit limit IN function, or terminal based, e.g. a prepaid SIM Card. Limiting risk also implies limiting access to high cost services such as International Dialling, International Diversion, Roaming.

\section{Diversion Fraud}

One of GSM's billing principles has the caller paying the standard charge irrespective of the handset location with the mobile owner paying the additional international charges when roaming internationally.

The fraud method uses a SIM, it is set for diversion to an overseas number so that the account owner pays the international charge. Although authentication back to the home operator occurs for each call the costs are charged back to the home operator each month. This method requires to be conducted in another country and it exploits the one month delay in billing, SIMs are obtained with a false identity or are stolen but still unreported.

The MSC provides all the switching function and when diversion is set the mobile number is released once the call is established. A new Diversion Number can be established while the other call(s) are in progress and so provides a mechanism for a call reselling 'business'. 
A typical sequence of events are:-

1) The handset sets a diversion to an international number (See Figure 1)

2) A call is made to the handset, the caller paying local call fee only, the handset owner pays for the cost of the diverted call, i.e. the international call costs. Nothing is paid if the call is made from a cloned analogue phone

3) Note the mobile side of the network is not involved and so this form of fraud is not detected by network congestion etc

4) The mobiles number is released once the call is established

5) While the first call is taking place the mobile sets the next diversion to another international number.

6) The next call is made to the mobile number. (See Figure 2)

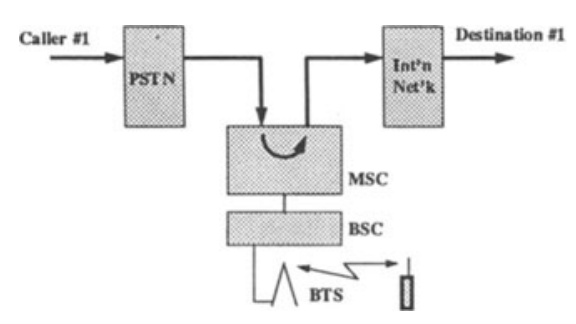

Figure 1

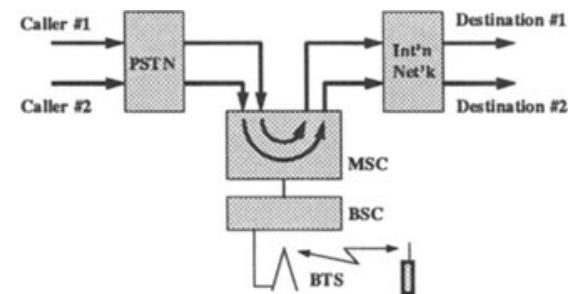

Figure 2

Diversion fraud can be detected by monitoring for simultaneous calls, long duration calls, call diversions being set/reset on a continuous basis. However reacting to this has the potential to upset legitimate customers. It is important to know how to handle customer liaison, if handled correctly it can be a bonus.

As most customers stay within their home country few need international roaming BUT even less need International Call Forward. Therefore to prevent this method the operator should separately block and release International Roaming from International Call Forward.

\section{Roaming - Fraud}

This is based on obtaining a SIM which is either stolen or obtained using subscription fraud. The SIM is obtained in one country and used in another; or used on another network within the same country if intercarrier roaming is available. It relies on the long bill reconciliation time (30+ days) even if "Home" operator has credit limiting in place.

It can be detected by speed up the loop between calls being made and charging information being passed back to the home operator. GSM is reducing the occurrence by:-

- Daily processing rather than end of month processing of charging records. The MoU now has set a maximum of 72 hours for the forwarding of charging records and this will be reduced to 36 hours by Sept ' 96 .

- Daily processing of accounts above a threshold, Fax or EDI notification to home operator. MoU recommend this to be approximately $\$ 150$. 
- Real Time processing rather than end of day/month batch processing. Many operators now will only set up a roaming agreement if online EDI reconciliation or twice a day EDI is available.

- Use an online Clearing House. The Clearing House only implements the notification, reconciliation as specified by the operator. It is up to the operator to establish a roaming agreement with all the necessary contractual requirements and then specify these to the Clearing House.

- To be effective operators need to move beyond a series of bilateral agreements.

- Build a relationship with other operators and develop a single and consistent agreement for charging and fraud management.

\section{Internal Fraud}

It can be caused by sales staff who are paid a commission for each new customer. They can invent customers and receive their sales commission. These 'customers' are easily detected as they don't make calls. One European operator reported that this accounted for $26 \%$ of all fraud and accounted for $7 \%$ of fraud losses.

Another method of internal fraud relates to activating a customer on the network by adding the customer directly to the network database, the same customer doesn't appear on the billing system. This can be prevented by activating all customers via the Billing systems including all test SIMs. In addition a comparison should be regularly run between the network databases and billing system to audit the procedure.

\section{Cloning Prevention}

Cloning is not a problem in GSM. To date there isn't evidence although there have been a number of claims. The industry investigates each very carefully and has found other causes rather than cloning. However if you don't look you won't see it should it happen!

If someone should clone a SIM by copying then they have cloned one card, they cannot guess any other cards IMSI and Ki combination from the knowledge gained.

Cloning in the analogue networks is detected by looking for multiple calls at one time or by Velocity Checking, here the handset cannot travel the distance between the two call locations with the time between calls. Artificial Intelligence systems are being introduced to detect the random calling nature of cloned handsets and finally systems are now going into service which monitor the unique RF 'fingerprint' of each mobile which determines if the handset is genuine or a clone.

\section{Resale of Subsidised Handsets}

When handsets are sold under a high subsidy environment, e.g. a purchase price of $\$ 100$ or less, there is the temptation to purchase the handset, pay the 12 months contract charge, make no calls and resell the handset. Due to the handset subsidy the handset is still valued in excess of the purchase price plus contract fee. Often the handset is purchased at a highly subsidised price and resold at wholesale price to another dealer, who may even claim subsidy again from the same operator! Network operators relying on usage to repay the subsidy. Is this illegal as the contract conditions have been met? 


\subsection{Conclusion on Fraud}

Although there are no reports of GSM been compromised, it is the increased functionality of the system that opens it to new forms of fraud. Fraud in a mobile environment also provides a new set of opportunities which do not occur in a fixed network. Roaming and the delays in inter operator charging have been exploited as one of the biggest opportunities for fraud.

Remember fraud will continue unless their profit margins can be reduced so it isn't a worthwhile business, alternatively fraud will decrease by reducing the convenience of their service. However it is important to balance this against the impact and inconvenience on genuine customers.

\section{HANDSET SECURITY}

As all customer information is contained in the SIM there potentially is no method to remotely to identify a handset. Stealing a handset and removing the SIM no longer identifies the handset in terms of the customer and the mobile number, therefore GSM has provided a handset electronic serial number, the International Mobile Equipment Identifier (IMEI).

During 1994 , the UK industry reported 12,000 were being stolen each month! Stolen handsets are an international trade and SIM sales without some check on the handset origin will only encourage this market. Handsets prices are usually subsidised by the network operator, in Australia we have recently seen handsets being offered for $\$ 9$. Replacing stolen and subsidised handsets creates a problem for the operator; Who replaces the handset? Can the customer afford a full price replacement handset? Is the replacement also subsidised? The dilemma is that the replacements usually aren't subsidised but the subsidies exist to stimulate the market by overcome the entry barrier of handset cost. To overcome this the UK operator 'Orange' includes handset insurance in the monthly service fee.

Customer service centres must also provide fast deactivation once a customer reports a handset stolen. Some network operators require a PIN code before a customer care centre will make any changes, i.e. caller authentication, and without the PIN code the service won't be deactivated. There has been the case where a customer rings the customer service centre to report a handset stolen but can't remember their PIN code. "I'm sorry we can't deactivate your service without your code", comes the response. It should be remembered that people forget codes and this needs to be built into the system, one method that can help is to not only record the customers pin code but also a prompt that will help them remember the code. The operator then says the prompt in the form of a question and the customer replies with the PIN code.

To manage the IMEI information each network can optionally establish an Equipment Identity Register (EIR) which has three potential categories. The White list is all satisfactory handsets which meet Type Approval, the Grey list which contains doubtful handsets that should be tested and repaired as they exceed the technical specifications and the Black list contains the IMEI of handsets reported stolen. 
The global EIR is planned to be established in Ireland, each operator optionally can run their own EIR with an data maintenance procedure providing the appropriate updates. If an operator runs an EIR its another database which needs maintenance, it incurs costs and it is questionable whether it returns a benefit. There have been cases where handsets on the Black list are not blocked but the customer details are passed to the Police for investigation. Thailand has an EIR, although the details are vague it is understood it only allows service to those handsets sold by the operator, i.e. if an operator makes money from handset sales, as opposed to a subsidy, then they want to provide an incentive for customers to purchase handsets from the operator!

\section{ALGORITHMS}

Three encryption algorithms are used within GSM, these are known as the A3, A5 and A8 algorithms.

A3 algorithm is used to Authenticate the SIM and therefore the customer.

A8 is used to deliver the key for use of the Air Interface algorithm.

A5 is used to encrypt the Air Interface.

The A3 and A8 algorithm are contained within the SIM card and are therefore developed and distributed at the discretion of the network operator. The A5 algorithm is installed in the handset and the Base Transceiver Station (BTS).

\subsection{Call establishment process}

Each customer is identified by their International Mobile Subscriber Identity (IMSI) number which is contained in their SIM card. When a call is attempted the handset forwards the IMSI to the home operator's Authentication Centre, the IMSI contains both the home operator's identity plus the customers identity. See Figure 3.

The home operator's Authentication Centre queries its data base to obtain that customer's Authentication Algorithm Key (Ki). It then generates a Random Number (RAND) which is sent to the handset transparently via the home operator's Mobile Switching Centre (MSC), the MSC of the host network, the Base Station Controller (BSC), Base Transceiver Station (BTS) and the Mobile Equipment (ME) (the handset), which in tern forwards it to the SIM card. It is the SIM which computes the Signed Response (SRES) which it sent back as far as the roaming network's MSC.

Meanwhile the home network's Authentication Centre has been computing the Signed Response for the handset using the same Ki. This is forwarded to the host operator's MSC to check against the mobiles SRES. If they are equal the call establishment process proceeds, if not the call is terminated.

If successful the voice traffic will be encrypted using the A5 algorithm, the key for this must be delivered to both the handset and the BTS. Delivery of this A5 key $(\mathrm{Kc})$ is performed by the A8 algorithm which is installed in the SIM. Kc is also calculated by the home network and 
delivered to the host network for use in the host network's BTS. The full key for the A5 algorithm is a combination of the Key Kc and the frame number of the radio link frame (n).

The home operator on receipt of request from a host network, simultaneously forwards RAND, SRES and Kc, which is known as a triplet. As the home operator and the host network may be some distance apart and due to transmission delays, the home network forwards three triplets to allow subsequent calls to be authenticated rapidly with the forwarding of replacement triplets being not as time critical.

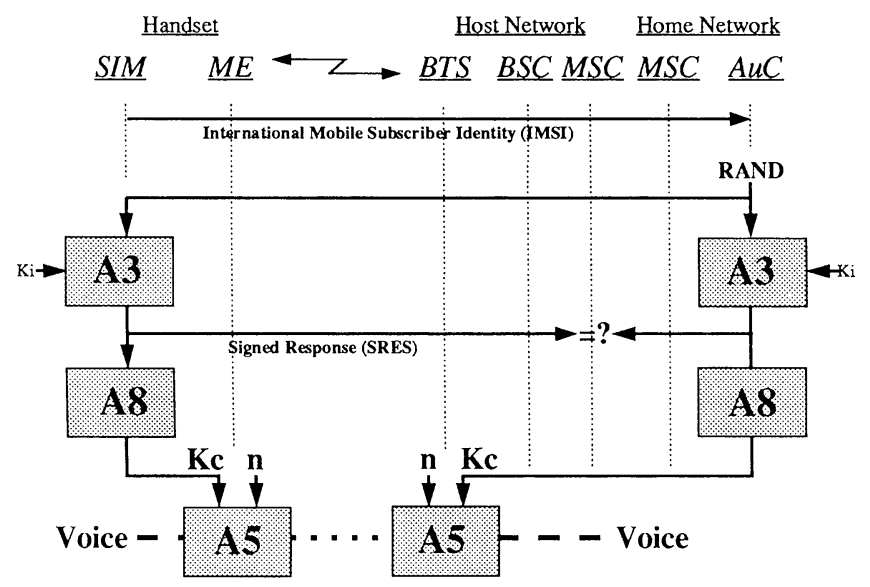

Figure 3 GSM Encryption Algorithms

\subsection{A3, A8 Algorithms}

Cloning and authentication is far more sensitive than eavesdropping and so although the A5 algorithm is shared the management of a single authentication algorithm was seen as being too difficult. If a single algorithm was used the consequences of a broken algorithm would allow all mobiles to be cloned as has happened in AMPS. The technique employed in GSM allows each operator to choose an algorithm, if one should be broken then other networks haven't been compromised. It also allows replacement algorithms to be implemented without requiring network changes.

RAND is 128 bits long, SRES must be 32 bits and $\mathrm{Ki}$ is any format and length as is the choice of A3/A8 at the discretion of the network operator.

The complexity of the A3 and A8 algorithms are limited by the memory capacity and processing speed of the Smart Card and so compromises are made on the level of security it provides. This when added to the limited distribution, ability to change at the desire of the 
operator allows GSM to continue to be a secure network. Each operator can select their own version of the A3 and A8 algorithm as they issue the SIM card to a customer.

Further to protect the anonymity of the mobile user once they first register with a system (by using their IMSI) they are issued a Temporary Mobile Subscriber Identity (TMSI) which replaces the IMSI.

\subsection{A5 Algorithm}

The algorithm is owned by the GSM MoU and is tightly copyright protected. Its interface requirements however are public and it works by taking a 22 bit Frame Number (n) from the air interface and a 64 bit key $(\mathrm{Kc})$ to produce a 114 bit sequence which is then Exclusively Or with the voice data stream. Two 114 bits streams are produced one to decode in the incoming voice and the other to encode the outgoing voice streams.

\section{Versions A5(1) \& A5(2)}

Two different versions of the A5 algorithm exist, the original A5(1) and a later version A5(2) was developed to meet the export licensing conditions of some countries. The need arose for two versions as GSM was originally designed for use in Western Europe and licensing did not allow for export outside this region. The rules for use are: countries who are full members of CEPT use A5(1) and all others use A5(2). Future handsets built to Phase 2 specifications will contain both A5(1) and A5(2) algorithms and will select the appropriate algorithm used in each country to allow for free circulation of handsets throughout the world. Interim use of A5(1) is allowed in some countries until the handsets are available which use A5(2) otherwise the air interface can work in clear mode.

The GSM MoU has a policy to establish a single algorithm for use worldwide.

\section{Activation process}

To allow for multiple air interface encryption algorithms and also where regulation prohibits the use of encryption the air interface starts in the clear mode and moves to the cipher mode in a managed way. This allows for handsets with incompatible A5 algorithms to make calls in the clear mode when roaming.

To move from clear mode to cipher mode is a three step process with the handset and BTS starting the communication process in clear mode. The BTS instructs the mobile to start sending on the uplink in encrypted mode, if it cannot decipher the stream it instructs the mobile to move back to the clear mode. When it has established communication on the uplink (mobile to BTS) in the ciphered mode the BTS notifies the mobile it is about to move the down link to the ciphered mode. The mobile reports back to the BTS if it can successfully decode the cipher stream otherwise the BTS reverts the downlink back to clear mode.

The cipher mode is established well before voice or data traffic starts and it occurs part way through the call set up process when only signalling information is being sent. To save attempting this process for all incompatible handsets each handset first sends a list of its capabilities via its Mobile Station Classmark code which includes details on revision level, power capability, A5 algorithm type, etc. 


\section{FUTURE DEVELOPMENTS}

As detection systems become more sophisticated and eliminate external fraud attacks internal fraud will rise as a proportion of total fraud and so we will see a focus on internal fraud.

The development of Dual Mode handsets, e.g. AMPS/GSM mainly to allow wider roaming using a single handset will open GSM users to the weaknesses of the other system, e.g. GSM users will be exposed to AMPS cloning fraud.

The biggest unknown is the potential for Value Added Services (VAS) to provide opportunities and weaknesses for exploitation. History has shown the more sophisticated the service the more sophisticated the form of fraud and the harder it is to detect. With so many VAS being considered and the ability to build and implement a VAS rapidly there is a big temptation to implement a VAS in response to a competitor without the due design process which includes a fraud risk assessment.

Whatever direction the technology goes, there is a bright future for people working in the area of security and risk assessment. 